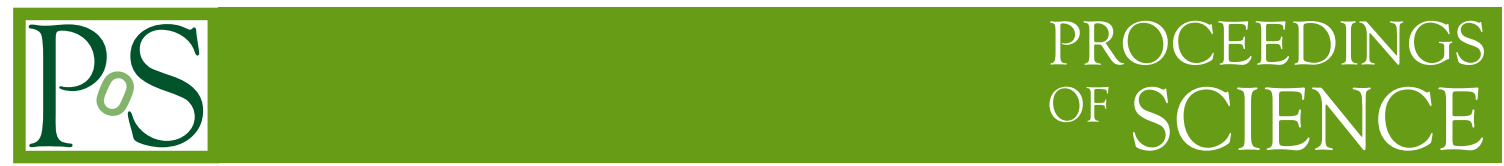

\title{
High-precision theory for top-quark production
}

\author{
Nikolaos Kidonakis ${ }^{* \dagger}$ \\ Department of Physics, Kennesaw State University, Kennesaw, GA 30144, USA \\ E-mail: nkidonak@kennesaw.edu
}

I present high-precision results for top quark production at hadron collider energies. Total and differential cross sections are calculated through $\mathrm{aN}^{3} \mathrm{LO}$ for top-antitop pair production and through aNNLO for single-top production. Top production in association with a charged Higgs boson and via anomalous couplings is also briefly discussed.

XIII International Conference on Heavy Quarks and Leptons

22-27 May, 2016

Blacksburg, Virginia, USA

\footnotetext{
*Speaker.

${ }^{\dagger}$ This material is based upon work supported by the National Science Foundation under Grant No. PHY 1519606.
} 


\section{Introduction}

The top quark is the heaviest elementary particle ever discovered and thus holds a very special place in the list of fundamental particles. It is predominantly produced in hadron colliders via top-antitop pair production processes and to a lesser extent via single-top production channels (for recent reviews see [1, 2]). Associated top production with a charged Higgs boson and top production via anomalous couplings are also of interest.

The QCD corrections to top production processes are typically quite large and need to be included for precise predictions. At current collider energies these corrections are dominated by soft-gluon emission. Thus one can derive excellent approximations to high-order corrections by calculating soft-gluon corrections. Such corrections need to be calculated through $\mathrm{N}^{3} \mathrm{LO}$ to achieve percent-level or better precision.

In this paper we present high-precision theoretical results for top quark total and differential cross sections in various processes, including top-antitop pair production $[3,4,5]$ in Section 2, single-top production $[6,7,8,9,10]$ in Section 3, top production in association with a charged Higgs boson [11] in Section 4, and top production via anomalous couplings [12] in Section 5.

\section{Top-antitop pair production}

The QCD corrections for $t \bar{t}$ production are quite large. Fixed-order NNLO calculations are now available based on a variety of analytical and numerical approaches by many groups (see $[2,13]$ for reviews). Soft-gluon corrections are dominant $[3,4,5]$ and they approximate the exact results very well at both NLO and NNLO.

We can calculate/resum these soft corrections at NNLL accuracy. Various approaches have been proposed over the years (see [13] for a review) and there are big differences in the accuracies of the various approximations. The method we use here has been the most successful in predicting the NNLO results, and the calculations of additional soft-gluon $\mathrm{N}^{3} \mathrm{LO}$ corrections from NNLL resummation provide the best theoretical predictions for the production cross sections and differential distributions $[3,4,5]$.

Approximate $\mathrm{N}^{3} \mathrm{LO}\left(\mathrm{aN}^{3} \mathrm{LO}\right)$ predictions for the $t \bar{t}$ cross section are derived by adding the third-order soft-gluon corrections to the fixed-order results:

$\mathrm{aN}^{3} \mathrm{LO}=\mathrm{LO}+\mathrm{NLO}+\mathrm{NNLO}+$ soft-gluon $\mathrm{N}^{3} \mathrm{LO}$ corrections

\subsection{Top-antitop pair $\mathrm{aN}^{3} \mathrm{LO}$ cross sections at the $\mathrm{LHC}$ and the Tevatron}

In Fig. 1 we plot the $\mathrm{aN}^{3} \mathrm{LO}[3] t \bar{t}$ total cross section with theoretical uncertainties at Tevatron and LHC energies and compare with recent data. The left plot shows results for the cross section at $1.96 \mathrm{TeV}$ Tevatron energy as a function of top quark mass while the plot on the right shows the cross section as a function of the LHC energy. The agreement between data and the $\mathrm{aN}^{3} \mathrm{LO}$ predictions is excellent in all cases.

The $\mathrm{aN}^{3} \mathrm{LO}$ total $t \bar{t}$ cross section with $m_{t}=173.3 \mathrm{GeV}$ and MSTW2008 NNLO pdf [14] takes the value of $810_{-16-32}^{+24+30} \mathrm{pb}$ at $13 \mathrm{TeV}$ LHC energy, where the first uncertainty is from scale variation and the second is the pdf uncertainty at $90 \%$ CL. 

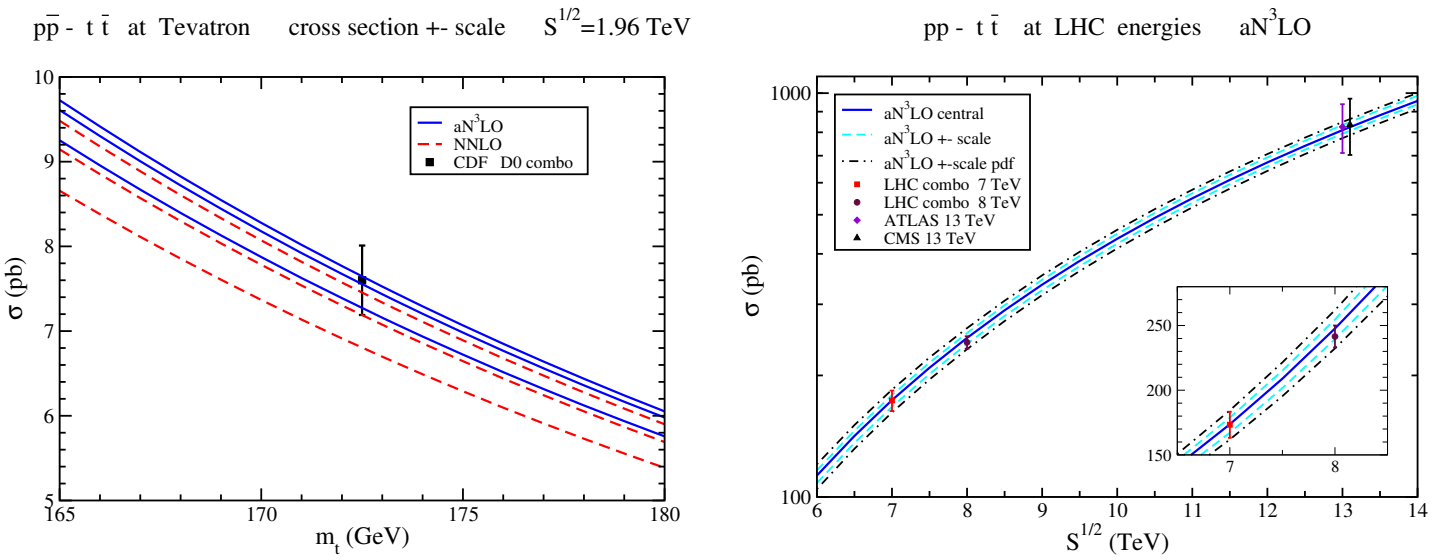

Figure 1: Top-antitop $\mathrm{aN}^{3} \mathrm{LO}$ cross sections at (left) the Tevatron compared with combined data from the CDF and D0 collaborations at $1.96 \mathrm{TeV}$ energy [15]; and (right) the LHC compared with combined data from ATLAS and CMS at $7 \mathrm{TeV}$ [16] and $8 \mathrm{TeV}$ [17], and with the new data at $13 \mathrm{TeV}$ from ATLAS [18] and CMS [19].

\subsection{Relative size of the perturbative corrections}

The relative sizes of the higher-order corrections and the convergence of the perturbative series are of course of great interest. We write the perturbative series through third order in the strong coupling as

$$
\sigma^{\mathrm{aN} \mathrm{N}^{3} \mathrm{LO}}=\sigma^{(0)}\left[1+\frac{\sigma^{(1)}}{\sigma^{(0)}}+\frac{\sigma^{(2)}}{\sigma^{(0)}}+\frac{\sigma^{(\mathrm{a} 3)}}{\sigma^{(0)}}\right]
$$

where $\sigma^{(0)}$ is the LO cross section, $\sigma^{(1)}$ and $\sigma^{(2)}$ are respectively the complete NLO and NNLO corrections, and $\sigma^{(\mathrm{a} 3)}$ are the $\mathrm{aN}^{3} \mathrm{LO}$ corrections.

\begin{tabular}{|c|c|c|c|c|c|}
\hline corrections & Tevatron $1.96 \mathrm{TeV}$ & LHC 7 TeV & LHC 8 TeV & LHC 13 TeV & LHC 14 TeV \\
\hline$\sigma^{(1)} / \sigma^{(0)}$ & 0.236 & 0.470 & 0.476 & 0.493 & 0.496 \\
\hline$\sigma^{(2)} / \sigma^{(0)}$ & 0.106 & 0.178 & 0.177 & 0.172 & 0.170 \\
\hline$\sigma^{(\mathrm{a} 3)} / \sigma^{(0)}$ & 0.068 & 0.066 & 0.059 & 0.045 & 0.043 \\
\hline$\sigma^{\mathrm{aN}^{3} \mathrm{LO}} / \sigma^{(0)}$ & 1.410 & 1.714 & 1.712 & 1.710 & 1.709 \\
\hline
\end{tabular}

Table 1: The fractional contributions to the perturbative series for the $t \bar{t}$ cross section.

In Table 1 we show the values of the ratios of the higher-order corrections to the LO cross section as well as the ratio - in the last line - of the total $\mathrm{aN}^{3} \mathrm{LO}$ cross section to the $\mathrm{LO}$ cross section. It is clear that higher-order corrections are very sizable for total cross sections, and this also holds for differential distributions. It is also obvious that NNLO is not enough! The $\mathrm{aN}^{3} \mathrm{LO}$ corrections are clearly needed for precision physics.

\subsection{Top-quark $\mathrm{aN}^{3} \mathrm{LO} p_{T}$ distributions at the LHC}

In Fig. 2 we plot the aN ${ }^{3} \mathrm{LO}$ [4] top-quark normalized transverse-momentum, $p_{T}$, distribution with scale variation at $7 \mathrm{TeV}$ LHC energy and compare with CMS data in the dilepton and 

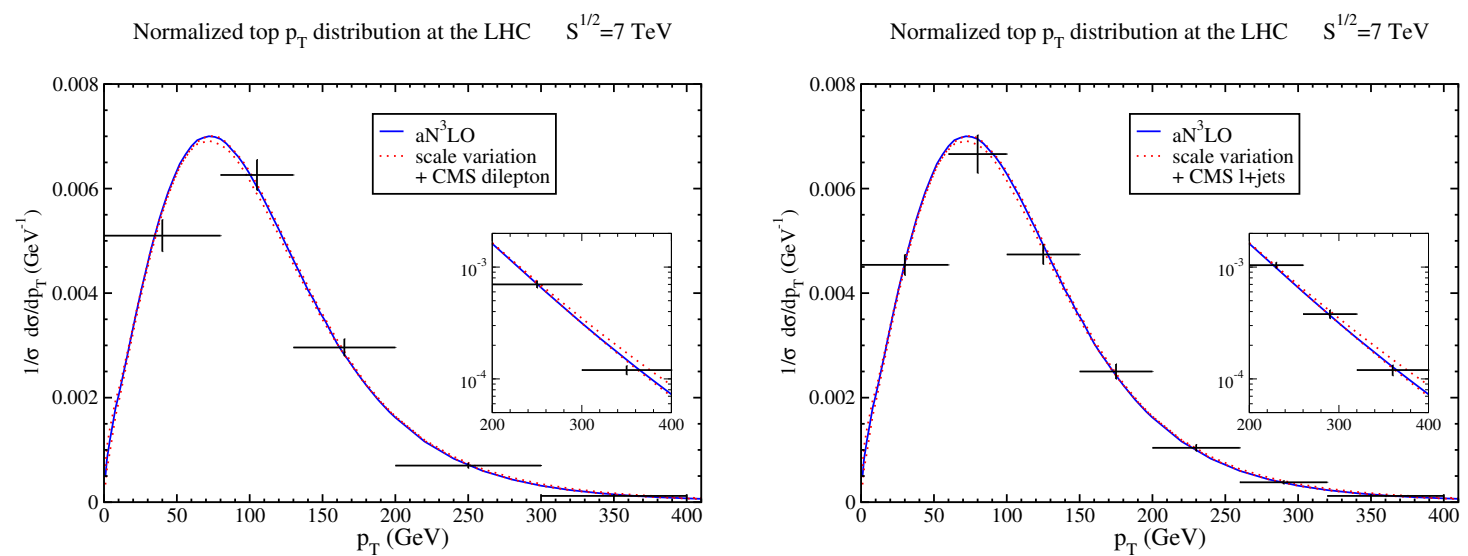

Figure 2: The $\mathrm{aN}^{3} \mathrm{LO}$ normalized top-quark $p_{T}$ distributions at $7 \mathrm{TeV}$ LHC energy compared with CMS dilepton (left) and lepton+jet (right) data [20].

lepton+jets channels, finding very good agreement in both cases. The inset plots highlight the large- $p_{T}$ region.
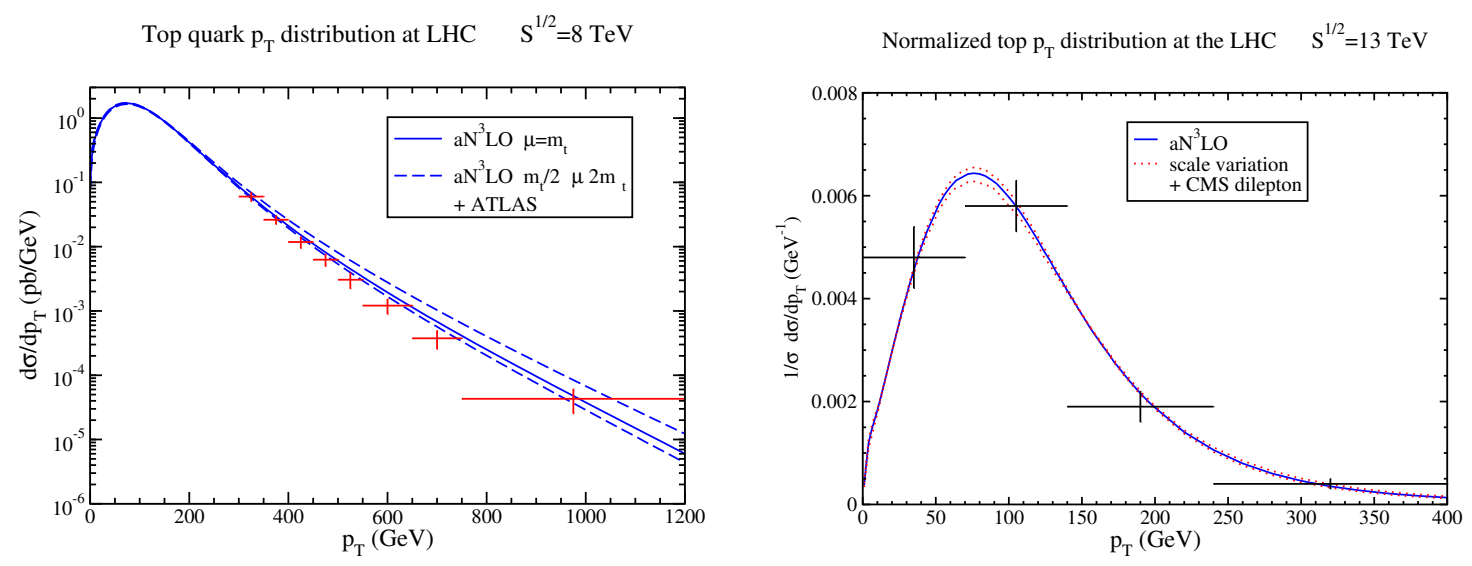

Figure 3: The $\mathrm{aN}^{3} \mathrm{LO}$ top-quark $p_{T}$ distribution (left) at the $8 \mathrm{TeV}$ LHC compared with ATLAS boosted-top data [21], and the normalized $p_{T}$ distribution (right) at the $13 \mathrm{TeV}$ LHC compared with CMS [22] data.

In the left plot of Fig. 3 we show the $\mathrm{aN}^{3} \mathrm{LO}$ [4] top-quark $p_{T}$ distribution at $8 \mathrm{TeV}$ LHC energy and compare with boosted-top data from ATLAS; the right plot shows the normalized top-quark $p_{T}$ distribution at $13 \mathrm{TeV}$ LHC energy compared with CMS data.

\subsection{Top-quark aN ${ }^{3} \mathrm{LO}$ rapidity distributions at the LHC}

In Fig. 4 we plot the $\mathrm{aN}^{3} \mathrm{LO}$ [4] top-quark normalized rapidity distribution at $7 \mathrm{TeV}$ LHC energy and compare with CMS data in the dilepton and lepton+jet channels, finding excellent agreement between theory and data in both channels.

In Fig. 5 we plot the $\mathrm{aN}^{3} \mathrm{LO}$ [4] top-quark normalized rapidity distribution at $8 \mathrm{TeV}$ (left plot) and $13 \mathrm{TeV}$ (right plot) LHC energies, and we compare with CMS data and find excellent agreement between theory and data at both energies. 

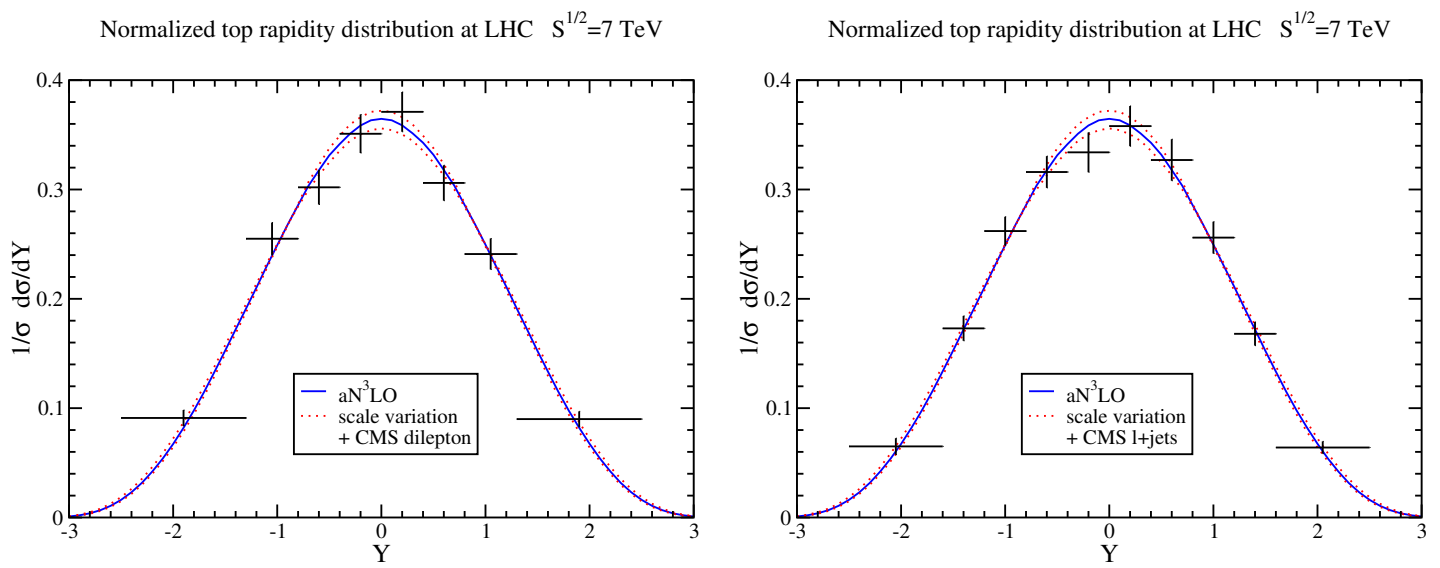

Figure 4: The $\mathrm{aN}^{3} \mathrm{LO}$ normalized top-quark rapidity distributions at $7 \mathrm{TeV}$ LHC energy compared with CMS dilepton (left) and lepton+jet (right) data [20].

Normalized top rapidity distribution at LHC $S^{1 / 2}=8 \mathrm{TeV}$

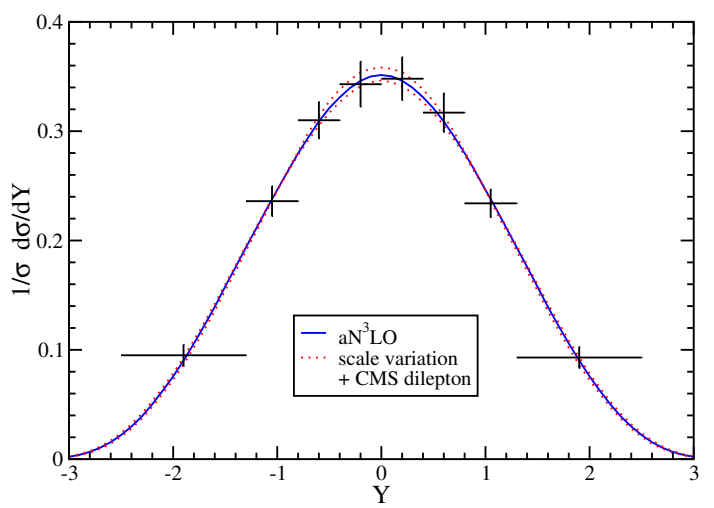

Normalized top rapidity distribution at LHC $\quad \mathrm{S}^{1 / 2}=13 \mathrm{TeV}$

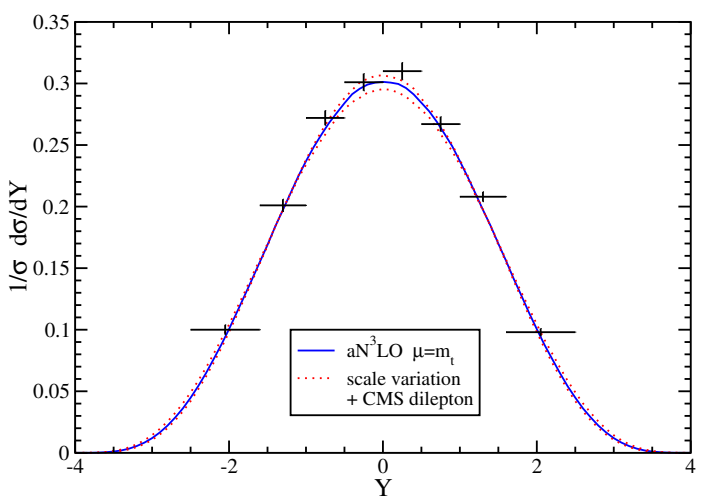

Figure 5: The $\mathrm{aN}^{3} \mathrm{LO}$ normalized top-quark rapidity distribution (left) at the $8 \mathrm{TeV}$ LHC compared with CMS data [23] and (right) at the 13 TeV LHC compared with CMS [24] data.

\subsection{Top-quark aN ${ }^{3} \mathrm{LO} p_{T}$ and rapidity distributions and $A_{\mathrm{FB}}$ at the Tevatron}

In the left plot of Fig. 6 we plot the $\mathrm{aN}^{3} \mathrm{LO}[4]$ top-quark $p_{T}$ distribution with scale variation at $1.96 \mathrm{TeV}$ Tevatron energy and find very good agreement with D0 data.

The top forward-backward asymmetry at the Tevatron, $A_{\mathrm{FB}}=\left[\sigma\left(y_{t}>0\right)-\sigma\left(y_{t}<0\right)\right] /\left[\sigma\left(y_{t}>\right.\right.$ $\left.0)+\sigma\left(y_{t}<0\right)\right]$, takes the $\mathrm{aN}^{3} \mathrm{LO}[5]$ QCD value -including EW corrections- of $(10.0 \pm 0.6) \%$ in the $t \bar{t}$ frame. We note that the corrections are large: the $\mathrm{aN}^{3} \mathrm{LO} / \mathrm{NNLO}$ ratio is 1.05 .

The top differential asymmetry $A_{\mathrm{FB}}^{\text {bin }}=\left[\sigma_{\text {bin }}^{+}(\Delta y)-\sigma_{\text {bin }}^{-}(\Delta y)\right] /\left[\sigma_{\text {bin }}^{+}(\Delta y)+\sigma_{\text {bin }}^{-}(\Delta y)\right]$, with $\Delta y=$ $y_{t}-y_{\bar{t}}$, is shown at $\mathrm{aN}^{3} \mathrm{LO}$ [5] in the right plot of Fig. 6 and compared to CDF and D0 data.

\section{Single-top production}

We continue with single-top production which can proceed through $t$-channel processes, $q b \rightarrow$ $q^{\prime} t$ and $\bar{q} b \rightarrow \bar{q}^{\prime} t ; s$-channel processes, $q \bar{q}^{\prime} \rightarrow \bar{b} t$; and associated $t W$ production, $b g \rightarrow t W^{-}$. 

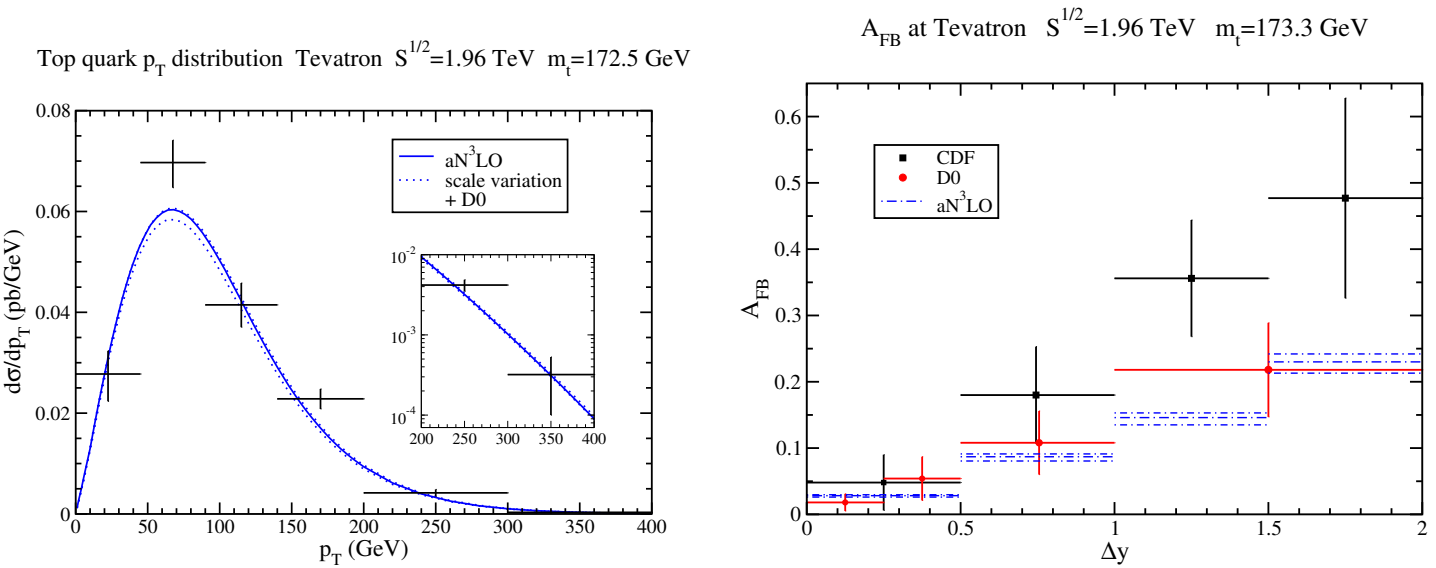

Figure 6: The $\mathrm{aN}^{3} \mathrm{LO}$ top-quark $p_{T}$ distribution at $1.96 \mathrm{TeV}$ Tevatron energy compared with D0 [25] data (left); and the $\mathrm{aN}^{3} \mathrm{LO}$ differential $A_{\mathrm{FB}}$ compared with CDF [26] and D0 [27] data.

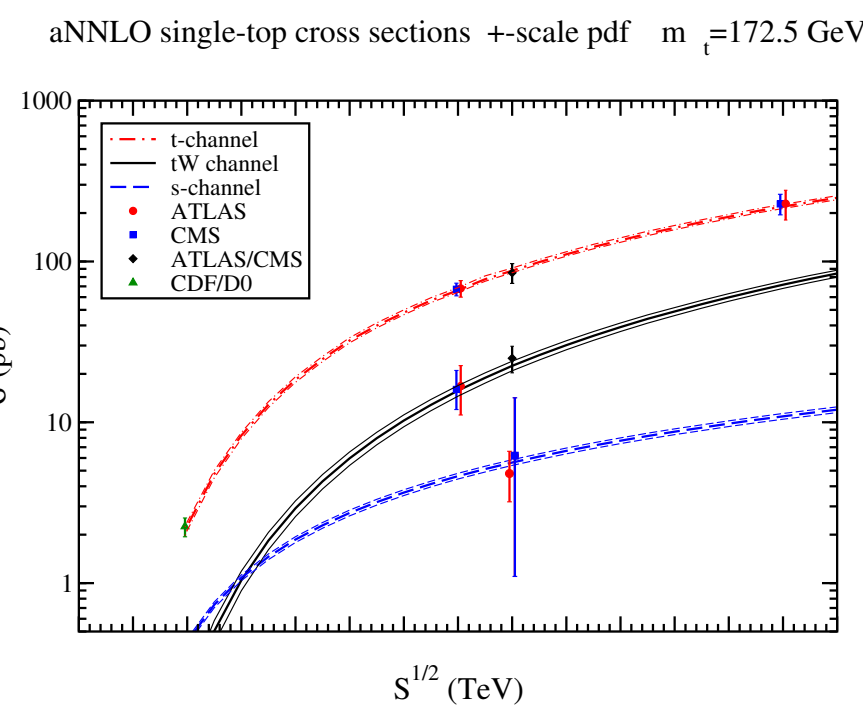

Figure 7: Single-top aNNLO cross sections at the LHC compared to $t$-channel data from CDF/D0 combination [28] at $1.96 \mathrm{TeV}$, ATLAS [29] and CMS [30] at $7 \mathrm{TeV}$, ATLAS/CMS combination [31] at $8 \mathrm{TeV}$, and ATLAS [32] and CMS [33] at $13 \mathrm{TeV}$; s-channel data from ATLAS [34] and CMS [35] at $8 \mathrm{TeV}$; and $t W$-channel data from ATLAS [36] and CMS [37] at $7 \mathrm{TeV}$, and ATLAS/CMS combination [38] at $8 \mathrm{TeV}$.

The aNNLO single-top cross sections as functions of LHC energy are shown in Fig. 7. As can be clearly seen, there is excellent agreement of theory $[6,7,8]$ with data for all three channels.

\subsection{Single-top $t$-channel production at aNNLO}

The aNNLO [8] single-top and single-antitop $t$-channel cross sections at the current $13 \mathrm{TeV}$ LHC energy with $m_{t}=173.3 \mathrm{GeV}$ are, respectively, $136_{-1}^{+3} \pm 3$ and $82_{-1}^{+2} \pm 2 \mathrm{pb}$. The errors indicated are from scale variation and pdf uncertainties from the MSTW2008 NNLO pdf at $90 \%$ CL. 
t-channel top $\mathrm{p}_{\mathrm{T}}$ distribution at LHC aNNLO $\mathrm{m}_{\mathrm{t}}=173.3 \mathrm{GeV}$

t-channel antitop $\mathrm{p}_{\mathrm{T}}$ distribution at LHC aNNLO $\mathrm{m}_{\mathrm{t}}=173.3 \mathrm{GeV}$
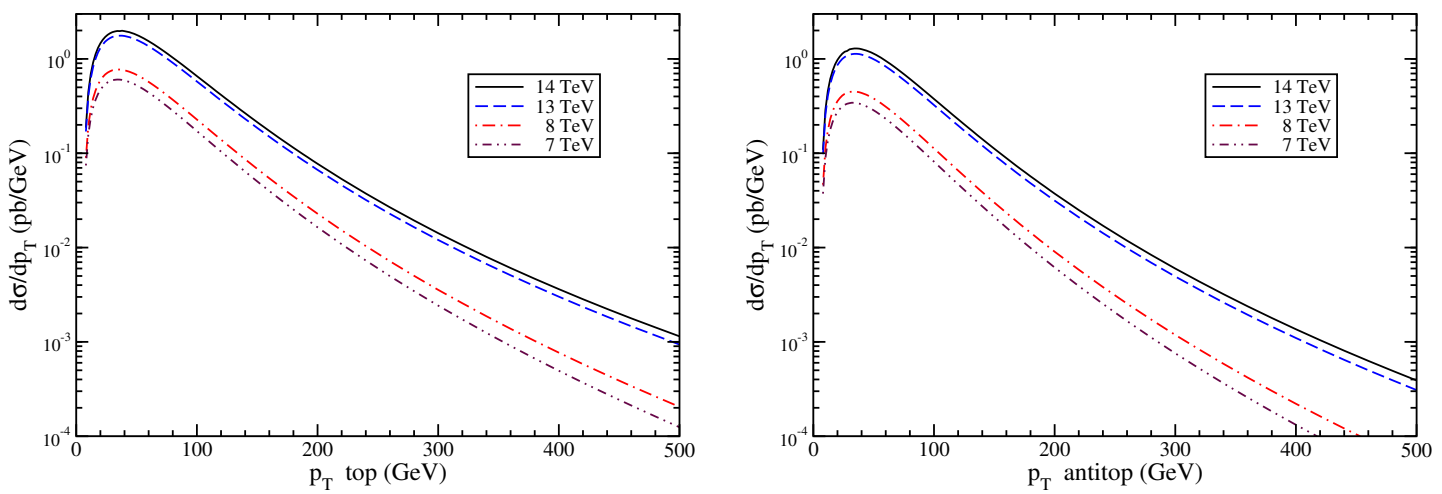

Figure 8: aNNLO $t$-channel top (left) and antitop (right) $p_{T}$ distributions at LHC energies.

The aNNLO $[9,10]$ top and antitop $p_{T}$ distributions in the $t$-channel at $7,8,13$, and $14 \mathrm{TeV}$ LHC energies are shown in Fig. 8.

$\mathrm{t}$-channel normalized top $\mathrm{p}_{\mathrm{T}}$ distribution at LHC aNNLO

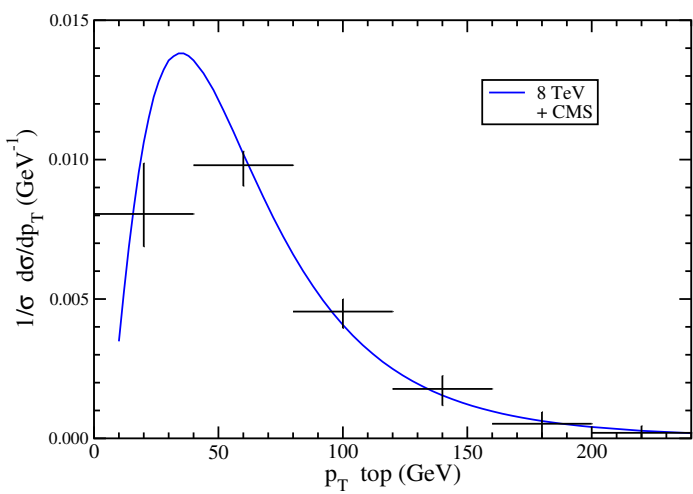

$\mathrm{t}$-channel normalized top+antitop $\mathrm{p}_{\mathrm{T}}$ distribution at LHC aNNLO

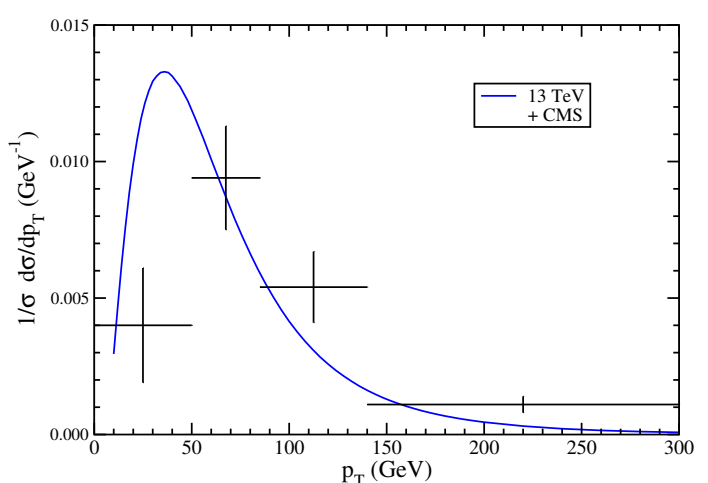

Figure 9: aNNLO $t$-channel normalized (left) top $p_{T}$ distribution compared with CMS data [39] at $8 \mathrm{TeV}$ and (right) top+antitop $p_{T}$ distribution compared with CMS data [40] at $13 \mathrm{TeV}$.

Some $t$-channel aNNLO normalized $p_{T}$ distributions at 8 and $13 \mathrm{TeV}$ energies at the LHC are shown in Fig. 9 and compared with CMS data.

\subsection{Single-top $s$-channel production at aNNLO}

The aNNLO [6] single-top and single-antitop s-channel cross sections at the current $13 \mathrm{TeV}$ LHC energy with $m_{t}=173.3 \mathrm{GeV}$ are, respectively, $7.07 \pm 0.13_{-0.22}^{+0.24}$ and $4.10 \pm 0.05_{-0.16}^{+0.14} \mathrm{pb}$.

The aNNLO [10] top and antitop $p_{T}$ distributions in the $s$-channel at 7, 8, 13, and $14 \mathrm{TeV}$ LHC energies and at $1.96 \mathrm{TeV}$ Tevatron energy are shown in Fig. 10.

\section{3 $t W^{-}$production at aNNLO}

The aNNLO [7] $t W^{-}$cross section at the current $13 \mathrm{TeV}$ LHC energy with $m_{t}=173.3 \mathrm{GeV}$ is $35.2 \pm 0.9_{-1.7}^{+1.6} \mathrm{pb}$. The cross section for $\bar{t} W^{+}$is the same. 
s-channel top $\mathrm{p}_{\mathrm{T}}$ distribution aNNLO $\mathrm{m}_{\mathrm{t}}=173.3 \mathrm{GeV}$

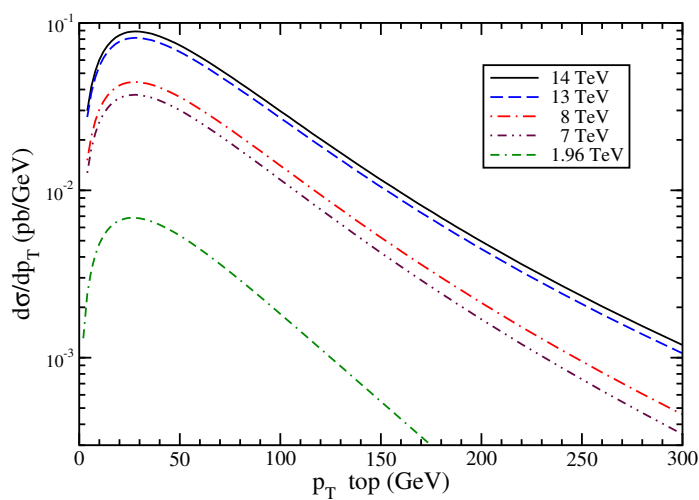

s-channel antitop $\mathrm{p}_{\mathrm{T}}$ distribution $\quad$ aNNLO $\quad \mathrm{m}_{\mathrm{t}}=173.3 \mathrm{GeV}$

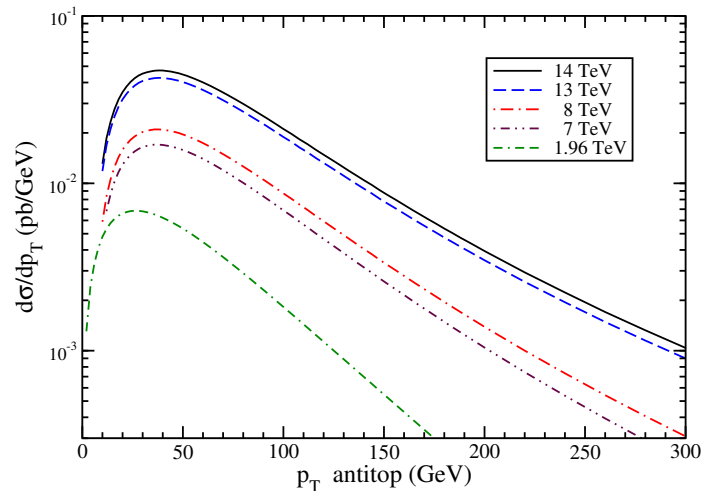

Figure 10: aNNLO $s$-channel top (left) and antitop (right) $p_{T}$ distributions at LHC and Tevatron energies.

Top $\mathrm{p}_{\mathrm{T}}$ distribution in $\mathrm{tW}^{-}$production at $\mathrm{LHC} \quad$ aNNLO $\mathrm{m}_{\mathrm{t}}=173.3 \mathrm{GeV}$

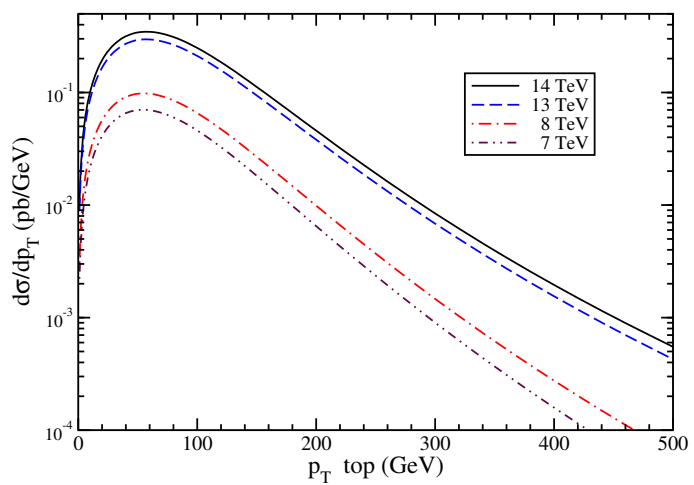

Top rapidity distribution in $\mathrm{tW}^{-}$production at $\mathrm{LHC}$ aNNLO $\mathrm{m}_{\mathrm{t}}=173.3 \mathrm{GeV}$



Figure 11: aNNLO top $p_{T}$ (left) and rapidity (right) distributions in $t W^{-}$production at LHC energies.

The aNNLO [10] top $p_{T}$ distributions in $t W^{-}$production at LHC energies are shown in the left plot of Fig. 11 together with new results for the rapidity distributions in the right plot.

\section{4. $t H^{-}$production at aNNLO}

We next present aNNLO [11] results for charged Higgs production in association with a top quark at LHC energies, using MMHT2014 NNLO pdf [41].

In Fig. 12 we plot the aNNLO [11] cross sections at various LHC energies as functions of the charged Higgs mass, and also the associated $K$ factors at $13 \mathrm{TeV}$ energy.

In Fig. 13 we plot the aNNLO [11] normalized top-quark $p_{T}$ and rapidity distributions at LHC energies for a charged Higgs mass of $300 \mathrm{GeV}$.

\section{Top production via anomalous couplings}

Single-top production may proceed via anomalous couplings in theories beyond the Standard Model. Soft-gluon corrections for top production via anomalous gluon couplings were calculated 

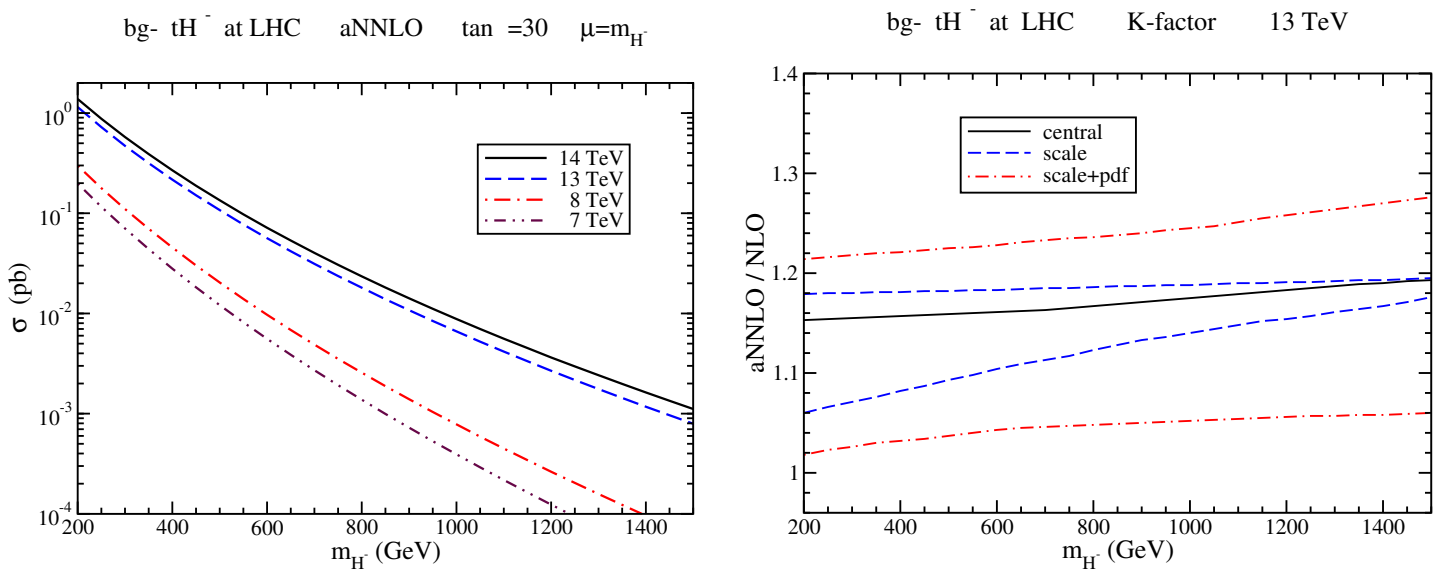

Figure 12: aNNLO cross sections at LHC energies (left) and $K$-factors at $13 \mathrm{TeV}$ (right) for $t H^{-}$production.
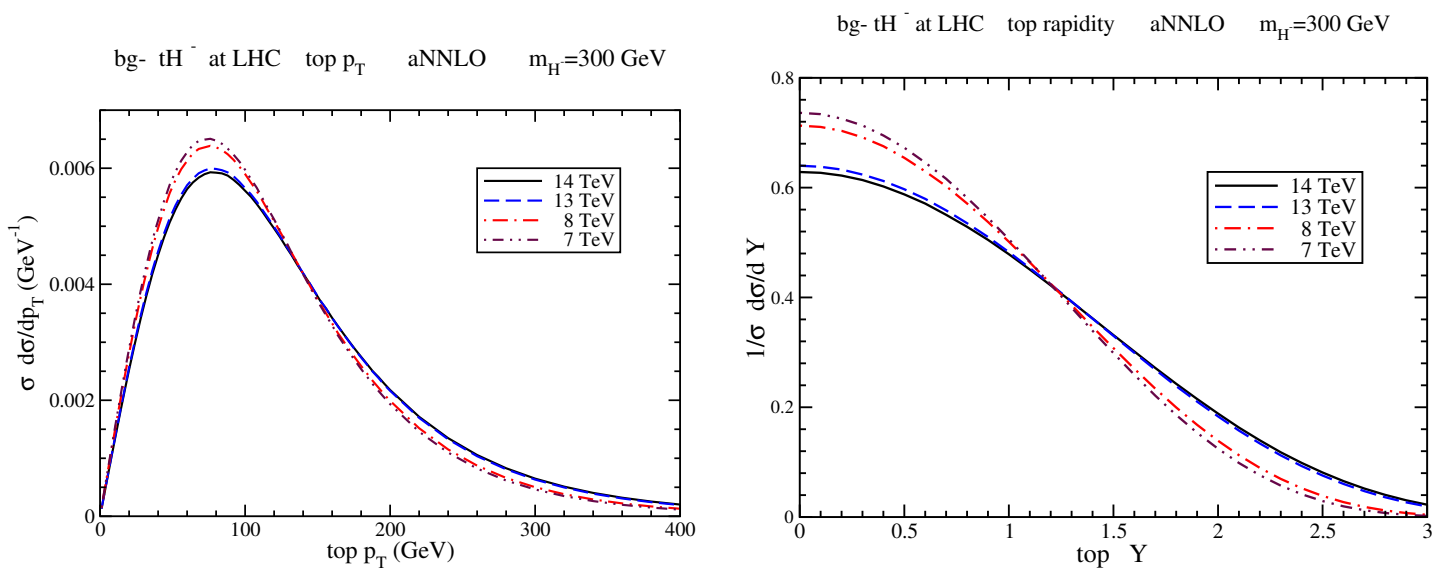

Figure 13: aNNLO top $p_{T}$ (left) and rapidity (right) distributions in $t H^{-}$production at LHC energies.

through aNNLO in [12]. For processes $g u \rightarrow t g$ via anomalous $t-u-g$ couplings, the soft-gluon corrections are significant in enhancing the cross section and reducing the scale dependence.

\section{References}

[1] J. Wagner-Kuhr, Experimental Studies of Top Quark Production, arXiv:1606.02936 [hep-ex].

[2] V. Del Duca and E. Laenen, Top physics at the LHC, Int. J. Mod. Phys. A 30, 1530063 (2015) [arXiv:1510.06690 [hep-ph]].

[3] N. Kidonakis, $N N N L O$ soft-gluon corrections for the top-antitop pair production cross section, Phys. Rev. D 90, 014006 (2014) [arXiv:1405.7046 [hep-ph]].

[4] N. Kidonakis, $N N N L O$ soft-gluon corrections for the top-quark $p_{T}$ and rapidity distributions, Phys. Rev. D 91, 031501(R) (2015) [arXiv:1411.2633 [hep-ph]].

[5] N. Kidonakis, The top quark forward-backward asymmetry at approximate $N^{3} L O$, Phys. Rev. D 91, 071502(R) (2015) [arXiv:1501.01581 [hep-ph]]. 
[6] N. Kidonakis, NNLL resummation for s-channel single top quark production, Phys. Rev. D 81, 054028 (2010) [arXiv:1001.5034 [hep-ph]].

[7] N. Kidonakis, Two-loop soft anomalous dimensions for single top quark associated production with a $W^{-}$or $H^{-}$, Phys. Rev. D 82, 054018 (2010) [arXiv:1005.4451 [hep-ph]].

[8] N. Kidonakis, Next-to-next-to-leading-order collinear and soft gluon corrections for t-channel single top quark production, Phys. Rev. D 83, 091503(R) (2011) [arXiv:1103.2792 [hep-ph]].

[9] N. Kidonakis, Top-quark transverse-momentum distributions in t-channel single-top production, Phys. Rev. D 88, 031504(R) (2013) [arXiv:1306.3592 [hep-ph]].

[10] N. Kidonakis, Single-top transverse-momentum distributions at approximate NNLO, Phys. Rev. D 93, 054022 (2016) [arXiv:1510.06361 [hep-ph]].

[11] N. Kidonakis, Charged Higgs production in association with a top quark at approximate NNLO, Phys. Rev. D 94, 014010 (2016) [arXiv:1605.00622 [hep-ph]].

[12] N. Kidonakis and E. Martin, Soft-Gluon Corrections in FCNC Top-Quark Production via Anomalous Gluon Couplings, Phys. Rev. D 90, 054021 (2014) [arXiv:1404.7488 [hep-ph]].

[13] N. Kidonakis and B.D. Pecjak, Top-quark production and QCD, Eur. Phys. J. C 72, 2084 (2012) [arXiv:1108.6063 [hep-ph]].

[14] A.D. Martin, W.J. Stirling, R.S. Thorne, and G. Watt, Parton distributions for the LHC, Eur. Phys. J. C 63, 189 (2009) [arXiv:0901.0002 [hep-ph]].

[15] CDF and D0 collaborations, Combination of measurements of the top-quark pair production cross section from the Tevatron Collider, Phys. Rev. D 89, 072001 (2014) [arXiv:1309.7570[hep-ex]].

[16] ATLAS and CMS Collaborations, Combination of ATLAS and CMS top-quark pair cross-section measurements using proton-proton collisions at $\sqrt{s}=7 \mathrm{TeV}$, ATLAS-CONF-2012-134, CMS PAS TOP-12-003.

[17] ATLAS and CMS Collaborations, Combination of ATLAS and CMS top quark pair cross section measurements in the e $\mu$ final state using proton-proton collisions at $\sqrt{s}=8 \mathrm{TeV}$, ATLAS-CONF-2014-054, CMS PAS TOP-14-016.

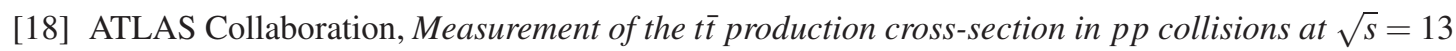
TeV using e $\mu$ events with b-tagged jets, ATLAS-CONF-2015-033.

[19] CMS Collaboration, Measurement of the inclusive and differential t $\bar{t}$ production cross sections in lepton + jets final states at $13 \mathrm{TeV}$, CMS-PAS-TOP-15-005.

[20] CMS Collaboration, Measurement of differential top-quark pair production cross sections in pp colisions at $\sqrt{s}=7$ TeV, Eur. Phys. J. C 73, 2339 (2013) [arXiv:1211.2220 [hep-ex]].

[21] ATLAS Collaboration, Measurement of the differential cross-section of highly boosted top quarks as a function of their transverse momentum in $\sqrt{s}=8 \mathrm{TeV}$ proton-proton collisions using the ATLAS detector, Phys. Rev. D 93, 032009 (2016) [arXiv:1510.03818 [hep-ex]].

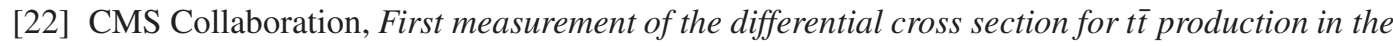
dilepton final state at $\sqrt{s}=13 \mathrm{TeV}$, CMS-PAS-TOP-15-010.

[23] CMS Collaboration, Measurement of the differential cross section for top quark pair production in pp collisions at $\sqrt{s}=8 \mathrm{TeV}$, Eur. Phys. J. C 75, 542 (2015) [arXiv:1505.04480 [hep-ex]].

[24] CMS Collaboration, Measurement of the differential cross section for $t \bar{t}$ production in the dilepton final state at $\sqrt{s}=13 \mathrm{TeV}$, CMS-PAS-TOP-16-011. 
[25] D0 Collaboration, Measurement of differential t $\bar{t}$ production cross sections in p $\bar{p}$ collisions, Phys. Rev. D 90, 092006 (2014) [arXiv:1401.5785 [hep-ex]].

[26] CDF Collaboration, Measurement of the top quark forward-backward production asymmetry and its dependence on event kinematic properties, Phys. Rev. D 87, 092002 (2013) [arXiv:1211.1003 [hep-ex]].

[27] D0 Collaboration, Measurement of the forward-backward asymmetry in top quark-antiquark production in p p collisions using the lepton+jets channel, Phys. Rev. D 90, 072011 (2014) [arXiv:1405.0421 [hep-ex]].

[28] CDF and D0 Collaborations, Tevatron combination of single-top-quark cross sections and determination of the magnitude of the Cabibbo-Kobayashi-Maskawa matrix element $V_{\mathrm{tb}}$, Phys. Rev. Lett. 115, 152003 (2015) [arXiv:1503.05027 [hep-ex]].

[29] ATLAS Collaboration, Comprehensive measurements of t-channel single top-quark production cross sections at $\sqrt{s}=7 \mathrm{TeV}$ with the ATLAS detector, Phys. Rev. D 90, 112006 (2014) [arXiv:1406.7844 [hep-ex]].

[30] CMS Collaboration, Measurement of the single-top-quark t-channel cross section in pp collisions at $\sqrt{s}=7 \mathrm{TeV}, J H E P$ 12, 035 (2012) [arXiv:1209.4533 [hep-ex]].

[31] ATLAS and CMS Collaborations, Combination of single top-quark cross-sections measurements in the $t$-channel at $\sqrt{s}=8 \mathrm{TeV}$ with the ATLAS and CMS experiments, ATLAS-CONF-2013-098, CMS-PAS-TOP-12-002.

[32] ATLAS Collaboration, Measurement of the inclusive cross-section of single top-quarkt-channel production in pp collisions at $\sqrt{s}=13 \mathrm{TeV}$, ATLAS-CONF-2015-079.

[33] CMS Collaboration, Measurement of the inclusive cross section of single top-quark production in the $t$-channel at $13 \mathrm{TeV}$, CMS-PAS-TOP-16-003.

[34] ATLAS Collaboration, Evidence for single top-quark production in the s-channel in proton-proton collisions at $\sqrt{s}=8 \mathrm{TeV}$ with the ATLAS detector using the Matrix Element Method, Phys. Lett. B 756, 228 (2016) [arXiv:1511.05980 [hep-ex]].

[35] CMS Collaboration, Measurement of the single top s-channel cross section at $8 \mathrm{TeV}$, CMS-PAS-TOP-13-009.

[36] ATLAS Collaboration, Evidence for the associated production of a $W$ boson and a top quark in ATLAS at $\sqrt{s}=7$ TeV, Phys. Lett. B 716, 142 (2012) [arXiv:1205.5764 [hep-ex]].

[37] CMS Collaboration, Evidence for associated production of a single top quark and $W$ boson in $\mathrm{pp}$ collisions at $\sqrt{s}=7 \mathrm{TeV}$, Phys. Rev. Lett. 110, 022003 (2013) [arXiv:1209.3489 [hep-ex]].

[38] CMS and ATLAS Collaborations, Combination of cross-section measurements for associated production of a single top-quark and $a \mathrm{~W}$ boson at $\sqrt{\mathrm{s}}=8 \mathrm{TeV}$ with the ATLAS and CMS experiments, CMS-PAS-TOP-14-009, ATLAS-CONF-2014-052.

[39] CMS Collaboration, Measurements of the differential cross section of single top-quark production in the $t$ channel in proton-proton collisions at $\sqrt{s}=8 \mathrm{TeV}$, CMS-PAS-TOP-14-004.

[40] CMS Collaboration, Measurement of the differential cross section for $t$-channel single-top-quark production at $\sqrt{s}=13 \mathrm{TeV}$, CMS-PAS-TOP-16-004.

[41] L.A. Harland-Lang, A.D. Martin, P. Molytinski, and R.S. Thorne, Parton distributions in the LHC era: MMHT 2014 PDFs, Eur. Phys. J. C 75, 204 (2015) [arXiv:1412.3989 [hep-ph]]. 